\title{
Evaluación de habilidades en matemática y comprensión lectora en estudiantes que ingresan a pedagogía en educación básica: un estudio comparativo en dos universidades del Consejo de Rectores ${ }^{1}$
}

\section{Math reasoning and reading comprehension skills assessment of entrant students from the primary school teaching program: a comparative study in two universities of the Council of Rectors (CRUCH)}

\author{
Pedro Rodrigo Sandoval Rubilar ${ }^{2}$ \\ Miguel Frit Carrillo ${ }^{3}$ \\ Ana Carolina Maldonado Fuentes ${ }^{4}$ \\ Francisco Rodríguez Alveal ${ }^{5}$
}

\begin{abstract}
RESUMEN
El artículo presenta los resultados de la evaluación realizada a estudiantes que ingresan a la carrera de Pedagogía en Educación General Básica, de dos instituciones universitarias regionales pertenecientes al Consejo de Rectores en Chile, en habilidades de razonamiento y conocimiento matemático y comprensión lectora. Corresponde a una evaluación diagnóstica de tipo descriptiva y comparativa de corte transversal, a una población compuesta por 80 alumnos, a quienes se les aplicó instrumentos debidamente validados con un Alfa de Cronbach $\alpha=0.82$ en Matemática y de $\alpha=0.64$ en Comprensión Lectora. Para
\end{abstract}

1 Departamento de Ciencias de la Educación, Facultad de Educación y Humanidades. Universidad del Bío-Bío. Avda. La Castilla, s/n, Casilla 447, Chillán, Chile.

2 Doctor en Ciencias de la Educación. Universidad del Bío-Bío, Chile. E-mail: psandoval@ ubiobio.cl.

3 Doctor en Educación Matemática. Universidad del Bío-Bío, Chile. E-mail: mfriz@ubiobio.cl.

4 Magíster en Educación mención Currículum y Evaluación. Universidad del Bío-Bío, Chile. E-mail: amaldonado@ubiobio.cl.

5 Magíster en Bioestadística. Universidad del Bío-Bío, Chile. E-mail: frodriguez@ubiobio.cl. 
el análisis de la información, se utilizaron métodos descriptivos univariados y multivariados. Los resultados evidencian, en general, un dominio descendido en ambas habilidades o por debajo de lo esperado al ingreso de la Educación Superior, especialmente en las habilidades matemáticas.

Palabras-clave: evaluación; formación inicial; comprensión lectora; habilidades matemáticas.

\begin{abstract}
Based on a comparative cross-assessment diagnosis conducted in two Council of Rectors (CRUCH) universities in southern Chile in 2009, this paper shows the results of Math reasoning and reading comprehension skills assessment of entrant students from the Primary School Teaching Program. Data for this paper comes from a test with Cronbach's Alpha reliability of 0,82 in Math and 0,64 in Reading Comprehension, which was applied to a sample of 80 students. Data analysis required one-variable and multivariable descriptive methods and the results dramatically showed either a low command of both skills or far lower than expected performance for entrant students from the Primary School Teaching Program, especially in Maths. Keywords: assessment; initial teaching preparation; reading comprehension; mathematical skills.
\end{abstract}

\title{
Antecedentes
}

En Chile, en el año 1996 se promulgó el Decreto Ley n. 40 (MINEDUC., 1996), que fijó nuevos Objetivos Fundamentales (OF) y Contenidos Mínimos Obligatorios $(\mathrm{CMO})$ para la Educación Básica, con lo cual se inició un proceso de renovación curricular en el Sistema Escolar Chileno, con el objeto de responder a las crecientes exigencias externas derivadas de la nueva sociedad del conocimiento y, al mismo tiempo, con la meta de subsanar carencias internas, como los bajos resultados en los aprendizajes de los estudiantes, particularmente en Educación Matemática y en Lenguaje y Comunicación, asunto que se evidenciaba especialmente en los sectores más pobres de la población (GARCÍA-HUIDOBRO; CÓX, 1999).

Esta carencia ha llevado al Gobierno de Chile a monitorear en forma permanente el estado de avance de las estrategias adoptadas en la Reforma; las cuales tienden o pretenden, entre otros puntos, mejorar la calidad de los logros obtenidos por los estudiantes, especialmente en Educación Matemática y Lenguaje y Comunicación.

Así pues, en 197, los alumnos de $3 .^{\circ}$ y $4 .^{\circ}$ años de Educación Básica participan en el Primer Estudio Internacional Comparativo en Lenguaje, Matemática 
y factores asociados, realizado por el Laboratorio Latinoamericano de Evaluación de Calidad de la Educación (LLECE, 1998), aplicado a un grupo de trece países. Dicha evaluación, en el área de Lenguaje, se centró en las competencias desarrolladas para comprender un texto; $\mathrm{y}$ en Matemáticas, la prueba midió las competencias para resolver problemas. Los resultados obtenidos mostraron bajos niveles de logro, en comparación al resto de los países. Del análisis general de este estudio se derivaron importantes sugerencias para las políticas educacionales, entre ellas: mejorar los aprendizajes en Lenguaje y Matemáticas, junto con favorecer la acción pedagógica efectiva, que conduzca a procesos de aprendizaje exitosos.

En el año 2000, los estudiantes que cursan entre Séptimo Básico y Cuarto Medio fueron sometidos a la evaluación del Program for International Student Assesment (PISA), que mide la capacidad para aplicar y relacionar conocimientos y habilidades en Lectura, Matemáticas y Ciencias.

En la evaluación de Lectura, el instrumento midió el nivel de comprensión, el uso y la reflexión sobre textos escritos, identificando para ello cinco niveles de desempeño. Los resultados muestran que los estudiantes chilenos, en promedio, tienen un rendimiento significativamente menor al promedio de los países de la Organización para la Cooperación y el Desarrollo Económico (OCDE). Las principales conclusiones de la evaluación permiten afirmar que nuestros estudiantes son capaces de realizar tareas básicas de lectura, hacer inferencias de baja complejidad, identificar la idea principal de un texto y hacer asociaciones sencillas entre el texto y su conocimiento previo.

Por su parte, la evaluación PISA en el área de Matemática midió la capacidad de identificar, comprender y plantear juicios bien fundamentados sobre el rol de las matemáticas en el mundo en la vida cotidiana. En promedio, los resultados obtenidos por los estudiantes chilenos los ubican en el nivel de habilidades más básicas. En términos operativos, esto significa que pueden completar procedimientos de un solo paso, como reproducir procesos matemáticos básicos o hacer cálculos simples. Asimismo, ellos pueden reconocer información de un texto familiar y directo en el cual la formulación matemática es explícita. Principalmente, lo que no pueden hacer es enfrentar problemas que requieren interpretar y formular un problema en términos matemáticos, generalmente en un contexto no familiar. Tampoco son capaces de manejar información compleja y hacer explícito un patrón o modelo matemático que puede estar o no en el problema.

De las conclusiones de la evaluación PISA (2000), se puede afirmar que nuestros estudiantes tendrían serias dificultades para usar la lectura como una herramienta efectiva para adquirir conocimientos y habilidades en otras áreas del saber, así como resolver problemas matemáticos que impliquen más de una operatoria o un razonamiento matemático que implique transferencia de conocimientos. 
Entre los años 1998 y 2002 a los alumnos de Educación Básica se les aplicó la evaluación TIMSS: Estudio Internacional de Tendencias en Matemáticas y Ciencias. Al rankear según su puntaje promedio, Chile se ubicó en el lugar 38 de 46 en Matemática, y 35 de 46 en Ciencias. Específicamente, en Matemáticas, más de la mitad de los alumnos chilenos (59\%) alcanzó el estándar de desempeño más bajo descrito por TIMSS, mostrando un dominio relativamente mejor en Estadística y Análisis de Datos, y más descendido en Geometría. En las conclusiones del estudio se señala que, en general, los docentes chilenos no tienen estudios de especialización en Matemáticas ni Ciencias (TIMSS, 2003).

Por otro lado, el Ministerio de Educación chileno ha promovido la evaluación de calidad de la educación a nivel nacional, a través del SIMCE (Sistema Nacional de Medición de la Calidad de la Educación), con el propósito de evaluar el logro de los Objetivos del Marco Curricular vigente para el Sistema Escolar Chileno. Especialmente mide el logro de aprendizaje de los estudiantes en tres momentos distintos durante su recorrido escolar (nivel cuarto y octavo básico, y segundo medio), en los Sectores de Lenguaje y Comunicación, Educación Matemática y Comprensión del Medio Natural, Social y Cultural.

Dicha evaluación, a partir del año 2008, informa los resultados según Niveles de Logro de los estudiantes: Inicial, Intermedio o Avanzado. Los resultados del año 2008 muestran que un 65\% de estudiantes alcanza el Nivel de Logro Intermedio o Avanzado y un 35\% se ubica en un Nivel Inicial. En Educación Matemática se observa que un 59\% de los alumnos se ubica en los Niveles de Logro Intermedio o Avanzado, y el $41 \%$ restante en la categoría Inicial (SIMCE, 2008).

Si bien, por una parte, los instrumentos y modelos de evaluación descritos no son homologables, y, por otro, las pruebas internacionales resultan más desconocidas por su reciente aplicación en nuestro sistema, no ocurre lo mismo con la prueba nacional SIMCE, dado que es heredera de una larga tradición de evaluación en el sistema escolar ${ }^{6}$. No obstante lo anterior, podemos afirmar que

6 Chile ha sido uno de los países pioneros en Latinoamérica en aplicar pruebas de rendimiento destinadas a medir logros de aprendizaje del sistema escolar, este tipo de pruebas datan de la Reforma Educacional de 1960, momento en el cual se aplicó la Prueba Nacional (1968) en 8. ${ }^{\circ}$ Básico con el propósito de aportar información para el desarrollo curricular y disponer de parámetros para mejorar la asignación de recursos. En 1982 surge el Programa de Evaluación del Rendimiento Escolar (PER) en $4 .^{\circ}$ y $8 .^{\circ}$ Básico, añadiendo la idea de poner en funcionamiento el sistema de voucher, con participación de padres y apoderados en la toma de decisiones de los establecimientos. Entre 1985 y 1986 el Centro de Perfeccionamiento, Experimentación e Investigaciones Pedagógicas (CPEIP) se hizo cargo del Sistema de Evaluación de la Calidad de la Educación (SECE) que retomaba los datos de la prueba PER. Desde 1988 está en vigencia el Sistema de Medición de la Calidad de la Educación SIMCE, aplicado en $4 .^{\circ}$ y $8 .^{\circ}$ Básico y II. ${ }^{\circ}$ Medio en las áreas de Lenguaje y Comunicación, Educación Matemática, Ciencias Naturales y Ciencias Sociales (EYZAGUIRRE; FONTAINE, 1999, p. 109-110). 
transcurrida más de una década de la última Reforma Educativa y Curricular, los niveles de logro alcanzados por nuestros estudiantes en los Sectores de Educación Matemática y Lenguaje y Comunicación no son los esperados por las políticas educativas impulsadas en dicho periodo, siendo los estudiantes de sectores socioeconómicos más bajos los que permanentemente muestran resultados bajos. Lo anterior, se sustenta en el hecho que independientemente del enfoque evaluativo adoptado por los instrumentos señalados anteriormente o del nivel de conocimiento de los mismos (tanto por parte de los profesores como de los estudiantes) la tendencia de los resultados de los logros de aprendizaje son en el tiempo bajos de manera persistente; en particular, en lo referido al dominio de la Comprensión Lectora y el Conocimiento y Razonamiento Matemático.

Por otro lado, se reconocen dimensiones positivas y negativas en este tipo de evaluaciones ${ }^{7}$; no obstante, los resultados han llevado a analizar el conjunto de sistema educativo para producir los cambios necesarios que permitan mejorar. Así pues, desde las políticas públicas del Estado se han impulsado una serie de transformaciones en el sistema y en el currículo escolar; además de impulsar una serie de programas; tales como: de apoyo pedagógico a los centros escolares, de mejoramiento de infraestructura, de recursos pedagógicos, incorporación de tecnologías a los establecimientos, entre otros. Esto significa asumir que existe un sinnúmero de factores que estarían incidiendo en los bajos resultados en las pruebas señaladas anteriormente.

En este contexto, en los últimos años, se ha puesto la atención en los profesores, especialmente lo referido a su preparación para enfrentar el nuevo currículo escolar. Dado que los estudios realizados sobre las evaluaciones nacionales han señalado que es posible obtener mejores resultados, independientes del tipo de establecimiento y nivel socioeconómico de los alumnos, si se cuenta con docentes con sólida formación disciplinaria y pedagógica (EYZAGUIRRE; FONTAINE, 2008).

Congruentemente, el Estado de Chile ha focalizado las políticas públicas en Educación en la Formación Inicial de los docentes, dado que sería una de las variables que explicaría en parte los resultados de los estudiantes del sistema escolar. De ahí que se postule la necesidad de contar con profesores competentes, que demuestren un buen desempeño en lo referido a los conocimientos y habilidades en Matemática y de Comprensión Lectora, puesto que los instru-

7 Entre los aspectos positivos se reconoce que las evaluaciones a nivel nacional permiten una retroalimentación a los establecimientos acerca de los aciertos y errores sobre las destrezas y los contenidos básicos de la enseñanza y aprendizaje. Como aspecto negativo, resalta el peligro de reducir la enseñanza sólo a lo que se evalúa, llevando a una ejercitación excesiva o entrenamiento puntual en determinados tipos de ítems. También se destaca el hecho de que las pruebas estandarizadas suelen tener un sesgo cultural al utilizar vocabulario propio del grupo social dominante (EYZAGUIRRE; FONTAINE, 1999). 
mentos de medición empleados en el sistema evalúan preponderantemente dichas habilidades. Así al menos lo sugiere el informe LLECE (1998; 2000), al constatar que existen diferencias de resultados de las escuelas según el estrato al que pertenecen, pero éstas desaparecen y, en algunos casos, se revierten, si el análisis se hace controlado por variables de los procesos educativos, como lo son la Formación Inicial del profesor, la capacitación y la experiencia docente.

Por lo mismo, el Gobierno de Chile, a partir del 2008 fortalece la idea de intervenir estructuralmente la Formación Inicial Docente en las universidades, con la finalidad de mejorar los estándares de aprendizaje de los profesionales que egresan de las Facultades de Educación del país, evaluando en forma diagnóstica los conocimientos fundamentales del currículo, incorporando las habilidades de Razonamiento Matemático y Comprensión Lectora (INICIA-MINEDUC., s/a).

En este contexto, el presente artículo pretende entregar información sobre el dominio de las Habilidades de Razonamiento y Conocimiento Matemático y Comprensión Lectora que poseen los estudiantes que ingresan a la Carrera de Pedagogía en Educación General Básica. La finalidad es reflexionar sobre el tipo de formación que deben recibir, así como de los contenidos y habilidades a desarrollar durante su proceso formativo.

\section{Problema de evaluación}

¿Cuál es dominio de las Habilidades de Razonamiento y Conocimiento Matemático y Comprensión Lectora que poseen los estudiantes que ingresan a Pedagogía en Educación General Básica?

\section{Objetivo general}

Diagnosticar el dominio en Habilidades de Razonamiento y Conocimiento Matemático y Comprensión Lectora que poseen estudiantes que ingresan a la Carrera Pedagogía en Educación General Básica, en dos Universidades del Consejo de Rectores de Chile.

\section{Objetivos específicos}

- Evaluar el dominio en Habilidades de Razonamiento y Conocimiento Matemático y Comprensión Lectora que poseen estudiantes que ingresan a la Carrera Pedagogía en Educación General Básica; 
- Identificar las relaciones entre los diferentes dominios medidos en las pruebas de Habilidades Matemáticas y Comprensión Lectora y los resultados obtenidos por los estudiantes de ingreso a la Carrera de Educación General Básica;

- Correlacionar los dominios de ambas pruebas, además de las variables de requisitos de ingreso (PSU) a la carrera en ambas universidades.

\section{Marco conceptual (variables evaluadas)}

\section{Razonamiento y conocimiento matemático}

Algunos estudios atribuyen los bajos resultados obtenidos por los estudiantes en pruebas de matemática, a que ciertos contenidos y objetivos del currículo no son enseñados en los tiempos definidos por el currículo, y otras veces lo son de manera tardía. Por su parte, para el Mineduc, el problema radica también en los aprendizajes claves que deben desarrollarse en este subsector los docentes del Sistema Escolar (QUIROZ, 1991; TIMSS, 2003).

Por lo anterior, el Mineduc ha elaborado los Mapas de Progreso y Ajustes Curriculares que pretenden explicitar las habilidades matemáticas centrales a ser aprendidas por los alumnos en los diferentes niveles del currículo escolar, los cuales se articulan en función de cuatro ejes; a saber: Forma y Espacio, Álgebra, Números, Datos y Azar (MINEDUC., 2007; 2009a).

Ahora bien, las habilidades matemáticas, según Llinares (2003), serían, entre otras: razonamiento matemático, comprensión conceptual (conocimiento) y destrezas procedimentales. Cada una de estas habilidades es compleja en sí misma y sus niveles de desarrollo están íntimamente ligados al aprendizaje progresivo de los sujetos, por ello se hace difícil evaluar o medir todas ellas en un solo instrumento.

Por lo anterior, el presente estudio se focalizará en las habilidades de Razonamiento y Conocimiento Matemático, dado que ambas están íntimamente ligadas al momento de enfrentar problemas matemáticos, puesto que es necesario comprender conceptualmente y al mismo tiempo razonar matemáticamente (identificar, inferir y reorganizar la información) para resolverlos (TIMSS, 2003). Para ello, se evaluarán las habilidades anteriormente mencionadas a través de los cuatro dominios que corresponden nominalmente a los ejes curriculares propuestos por el MIMEDUC (2007), que son: Forma y Espacio, Álgebra, Números, Datos y Azar, adaptando reactivos de la Prueba SIMCE. 


\section{Comprensión lectora}

Mejorar el dominio Lingüístico de los estudiantes, definida como capacidad para usar la lengua, es una de las funciones fundamentales del sector Lenguaje y Comunicación en la Educación Básica (MINEDUC, 2009b). Para esto, es necesario promover el desarrollo de cuatro habilidades comunicativas, a saber: expresión escrita, expresión oral, comprensión auditiva y comprensión lectora, tal como se explicita en los Mapas de Progreso y en los Ajustes Curriculares.

Evaluar cada una de las habilidades lingüísticas de una sola vez y en un solo instrumento se hace poco operativo y, por lo anterior, se tiende a hacerlo separadamente; siendo la Comprensión Lectora una de las competencias más medidas, independientemente del contenido disciplinario y la tipología textual de la que se trate. Por ello, se opta por evaluar la Habilidad de Comprensión Lectora.

En el presente estudio se adopta el Modelo Interactivo de la Lectura, citado por Catalá et. al. (2005), entendiendo que la Comprensión Lectora se da en el diálogo entre el texto y el lector, siendo este último quien aporta sus representaciones mentales, sus conocimientos acerca del mundo y sobre la lengua al momento de leer. Esto significa que para leer es necesario dominar las habilidades de decodificación y las estrategias necesarias para procesar activamente el texto, de modo tal que sea posible verificar las predicciones e hipótesis que se van formulando durante la lectura.

Desde esta perspectiva, es posible distinguir cuatro niveles de Comprensión Lectora, independientemente de que sean utilizados en forma simultánea durante el proceso lector. En esta evaluación, operativamente, dichos niveles equivalen a los siguientes dominios: Comprensión Literal, Reorganización de Información, Comprensión Inferencial y Comprensión Crítica.

\section{Diseño metodológico de la evaluación}

\section{Población o sujetos de estudio}

Los sujetos de estudio estuvo compuesto por 80 alumnos de la Carrera de Pedagogía en Educación General Básica, de ingreso 2009 en dos Universidades del Consejo de Rectores. En términos generales, el 37,5\% pertenecen a la Universidad n. 1 y el $62,5 \%$ a la Universidad n. 2 .

En relación a las variables de control, se puede señalar que el 100\% de ellos ingresa vía Prueba Selección Universitaria (PSU), de carácter nacional 
y obligatoria. Respecto a los puntajes de ingreso en la PSU Lenguaje y PSU Matemática, según se observa en la Tabla 1, los alumnos de la Universidad n. 2 ingresan con un puntaje promedio más alto que los de la Universidad n. 1 . En relación a la Desviación Estándar de los puntajes, en general existe una alta dispersión; sin embargo, en los puntajes de la PSU Matemática la variabilidad tiende a ser numéricamente similar.

Por otra parte, en relación a la variable procedencia, el $46 \%$ proviene de establecimientos educacionales Municipalizados, mientras que el $54 \%$ de establecimientos educacionales Particulares Subvencionados ${ }^{8}$.

TABLA 1 - ESTADÍSTICAS BÁSICAS SEGÚN UNIVERSIDAD DE PROCEDENCIA

\begin{tabular}{|c|c|c|c|c|c|}
\hline & & \multicolumn{2}{|c|}{ 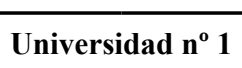 } & \multicolumn{2}{|c|}{ Universidad $n^{\circ} 2$} \\
\hline & & $\mathbf{N}$ & $\%$ & $\mathbf{N}$ & $\%$ \\
\hline \multirow{2}{*}{ Genero } & Femenino & 23 & 77 & 41 & 82 \\
\hline & Masculino & 7 & 23 & 9 & 18 \\
\hline \multirow{2}{*}{ Dependencia } & Municipal & 10 & 36 & 26 & 52 \\
\hline & Subvencionado & 18 & 64 & 24 & 48 \\
\hline \multirow{2}{*}{ PSU } & Lenguaje $^{1}$ & \multicolumn{2}{|c|}{$539,8 \pm 30,7$} & \multicolumn{2}{|c|}{$580,7 \pm 43,8$} \\
\hline & Matemática $^{1}$ & \multicolumn{2}{|c|}{$509,4 \pm 42,7$} & \multicolumn{2}{|c|}{$559,3 \pm 39,4$} \\
\hline
\end{tabular}

${ }^{1}:$ Promedio \pm Desviación Estándar

Se puede observar que mayoritariamente ingresan estudiantes de género femenino a estudiar la Carrera de Pedagogía en Educación General Básica en ambas universidades con porcentajes del orden del $77 \%$ y del $82 \%$, respectivamente. En la Universidad n. 1 el $64 \%$ de los estudiantes provienen de establecimientos Particulares Subvencionados, en cambio en la Universidad n. 2 los alumnos mayoritariamente son de establecimientos Municipalizados.

8 En el sistema escolar chileno los establecimientos se clasifican según el tipo de sostenedor: Municipal, Particular Subvencionado y Particular Pagado. Según los índices oficiales al año 2008, los establecimientos Municipales, que dependen del Estado, corresponden al 49\% del total de establecimientos del país; los Particulares Subvencionados, que dependen de un privado y son subvencionados por el Estado, corresponden a un 45\%; y, los establecimientos Particulares Pagados, que dependen de un privado y no tienen subvención del Estado, corresponden a un $6 \%$. (Fuente MINEDUC: $<$ http://w3app.mineduc.cl/DedPublico/educacion_en_cifras $>$ ). 
Por último, según el nivel socioeconómico, se trata de una muestra donde el $80 \%$ de los alumnos provienen de hogares clasificados en los tres primeros quintiles (I, II y III) que pertenecen a la población más vulnerable de Chile. Los quintiles contemplan el ingreso per cápita de los hogares del país, como se muestra en la siguiente tabla (EDUCARCHILE, 2010):

TABLA 2 - NIVEL SOCIOECONÓMICO

\begin{tabular}{c|l|c|c}
\hline QUINTILES & \multicolumn{1}{|c|}{ CLASIFICACIÓN } & US\$* & REAL** \\
\hline Quintil I & $\begin{array}{l}\text { Incluye a todas las familias cuyo ingreso per } \\
\text { cápita sea igual o inferior a \$39.990. }\end{array}$ & 72,78 & 137.591 \\
\hline Quintil II & $\begin{array}{l}\text { Corresponde a todas las familias cuyo ingre- } \\
\text { so per cápita sea igual o inferior a \$67.866. }\end{array}$ & 123,80 & 264.044 \\
\hline Quintil III & $\begin{array}{l}\text { Incluye a las familias cuyo ingreso per cápi- } \\
\text { ta sea igual o inferior a \$107.292. }\end{array}$ & 195,72 & 370.009 \\
\hline Quintil IV & $\begin{array}{l}\text { Corresponde a todas las familias cuyo ingre- } \\
\text { so per cápita sea igual o inferior a \$200.000. }\end{array}$ & 364,84 & 689.73 \\
\hline Quintil V & $\begin{array}{l}\text { Corresponde a todas las familias cuyo in- } \\
\text { greso per cápita sea superior a \$200.000. }\end{array}$ & 365.02 & 690.07 \\
\hline
\end{tabular}

*: Fuente: Banco Central de Chile, 24 de mayo. Dólar Observado \$ 548,18.

**: Fuente: Economía y Negocios, EMOL. 25 de mayo del 2010.

\section{Tipo de evaluación y análisis de datos}

Se trata de una evaluación diagnóstica de finalidad descriptiva comparativa, de corte transversal, ya que se aplica una sola vez, a estudiantes de Ingreso a la Carrera de Educación General Básica de dos universidades pertenecientes al Consejo de Rectores, entre los meses de marzo y mayo del año 2009 (COOK; REICHARDT, 1986; LATORRE, 1997).

Para la tabulación de la información se utilizaron métodos descriptivos tanto gráficos como numéricos de tendencia central (Porcentajes y Media Aritmética) y de variabilidad (Desviación Estándar); además, técnicas de análisis multivariado (JONSON; WICHERN, 1988; COLLINS, 1992; CRIVISQUI, 1993). El procesamiento de la información se realizó en el programa SPSS versión 11.0 para Windows, SPAD versión 5 y en el programa Excel.

La presente evaluación utilizó como referente principal el porcentaje (\%) de respuestas correctas obtenidas por los estudiantes del total de preguntas formuladas 
por cada ítem, según el domino de cada prueba. Además, se utiliza como referente el porcentaje (\%) promedio obtenido por el grupo e intragrupo. Este análisis se fundamenta dado que no existen estándares nacionales normalizados referidos a las habilidades de Razonamiento y Conocimiento Matemático y Comprensión Lectora, tanto para el ingreso como para el egreso de la Carrera de Pedagogía en Educación General Básica en Chile. Por tanto, una de las primeras sugerencias a las autoridades encargadas de diseñar las Política Publicas en Educación, especialmente las referidas a la Formación Inicial Docente y Habilitación para el ejercicio profesional, sería definir estándares mínimos normalizados.

\section{Instrumentos de evaluación}

La presente evaluación se basa en la aplicación de dos instrumentos de papel y lápiz de respuesta cerrada. El primero, de Habilidades de Razonamiento y Conocimiento Matemático se diseñó sobre la base de los cuatro ejes propuestos en el currículum escolar: Forma y Espacio, Álgebra, Números, Datos y Azar. Adaptando reactivos de la Prueba SIMCE ( $8 .^{\circ}$ básico) y, el segundo, de Habilidad de Comprensión Lectora, corresponde a una adaptación del las Pruebas ACL (CATALÁ et. al., 2005), que establece cuatro niveles: Comprensión Literal, Reorganización de Información, Comprensión Inferencial y Comprensión Crítica.

El instrumento de Habilidades de Razonamiento y Conocimiento Matemático tiene como finalidad constatar el logro de aprendizajes que se supone fueron adquiridos por parte de los estudiantes que ingresan a la Educación Superior, en el proceso de escolarización en Educación Matemática. Esta prueba consta de 28 ítems con un Alpha de Cronbach $\alpha=0.82$.

La prueba de Comprensión Lectora tiene por finalidad

valorar la comprensión lectora de una manera amplia, a partir de textos de tipología diversa, con temáticas referidas a diferentes áreas curriculares, con preguntas que recogen las dimensiones de comprensión: literal, reorganización, inferencial y crítica (CATALÁ et. al., 2005, p. 41).

El proceso de adaptación de las pruebas ACL se realizó seleccionando textos y reactivos, junto con efectuar ajustes idiomáticos en palabras que aludían a modismos, expresiones de uso diario y términos geográficos, de modo tal que se represente el español de Chile, en el nivel culto. El instrumento final consta de 30 ítems con un Alpha de Cronbach $\alpha=0.65$. 
RUBILAR, P. R. S.; CARRILLO, M. F.; FUENTES, A. C. M.; ALVEAL, F. R. Evaluación de habilidades...

TABLA 3 - ESPECIFICACIONES DE LOS INSTRUMENTOS DE EVALUACIÓN

\begin{tabular}{l|c}
\hline \multicolumn{2}{c}{ Prueba de Habilidades de } \\
Comprensión Lectora \\
\hline Dominios Evaluados & N. $^{\text {preguntas }}$ \\
\hline Comprensión Literal & 9 \\
\hline Comprensión Inferencial & 8 \\
\hline Comprensión Crítica & 9 \\
\hline Reorganización de Información & 4 \\
\hline TOTAL & $\mathbf{3 0}$ \\
\hline
\end{tabular}

\begin{tabular}{l|c}
\hline \multicolumn{2}{c}{$\begin{array}{c}\text { Prueba de Habilidades de } \\
\text { Matemático } \\
\text { Mazonamiento y Conocimiento }\end{array}$} \\
\hline Dominios Evaluados & $\begin{array}{c}\text { N. }^{\mathbf{2}} \\
\text { preguntas }\end{array}$ \\
\hline Números & 5 \\
\hline Álgebra & 7 \\
\hline Geometría & 8 \\
\hline Datos y Azar & 8 \\
\hline TOTAL & $\mathbf{2 8}$ \\
\hline
\end{tabular}

\section{Resultados}

Los resultados obtenidos de la aplicación de los instrumentos se presentarán en tres partes. En primer lugar, los porcentajes de logro obtenidos por los estudiantes en los dominios evaluados en ambas pruebas, junto a la variabilidad de los resultados según la universidad de procedencia. En segundo término, el análisis de correlación existente entre los diferentes dominios de cada una de las pruebas y entre las mismas. Finalmente, el análisis de correlación entre los dominios evaluados y la variable PSU.

\section{Resultados de logro en los dominios evaluados}

a) Prueba de Habilidades de Razonamiento y Conocimiento Matemático

En relación con los dominios evaluados en este instrumento, se observó que el mejor logrado corresponde a Números con un $82,8 \%$ promedio, siendo a su vez el que presenta menor variabilidad en comparación a los otros dominios medidos. Por su parte, el más descendido corresponde a Forma y Espacio (47,8\% promedio), presentando la mayor variabilidad en el porcentaje de respuestas (ver Tabla 4). 
RUBILAR, P. R. S.; CARRILLO, M. F.; FUENTES, A. C. M.; ALVEAL, F. R. Evaluación de habilidades...

TABLA 4 - PORCENTAJES DE LOGRO EN LOS DOMINIOS EVALUADOS SEGÚN INSTITUCIÓN PRUEBA DE HABILIDADES DE RAZONAMIENTO Y CONOCIMIENTO MATEMÁTICO

\begin{tabular}{l|c|c|c|c}
\hline \multicolumn{1}{c|}{ DOMINIO } & $\begin{array}{c}\text { Número } \\
\text { Preguntas }\end{array}$ & $\begin{array}{c}\text { Universidad 1 } \\
(\mathbf{n = 3 0 )}\end{array}$ & $\begin{array}{c}\text { Universidad 2 } \\
(\mathbf{n = 5 0 )}\end{array}$ & $\begin{array}{c}\text { Total } \\
(\mathbf{n = 8 0})\end{array}$ \\
\hline Datos y Azar & 6 & $47,0 \pm 20,7^{1}$ & $61,7 \pm 21,2$ & $52,7 \pm 22,0$ \\
\hline Números & 7 & $78,9 \pm 21,8$ & $88,9 \pm 15,1$ & $82,8 \pm 20,0$ \\
\hline Forma y Espacio & 6 & $40,6 \pm 26,2$ & $59,4 \pm 26,4$ & $47,8 \pm 27,7$ \\
\hline Algebra & 6 & $49,1 \pm 19,6$ & $66,4 \pm 23,3$ & $55,6 \pm 22,6$ \\
\hline
\end{tabular}

1: Promedio \pm Desviación Estándar (D.E).

La Figura 1 corresponde a una representación del análisis de la distribución general de los porcentajes de respuestas correctas obtenidas por el total de la población, en cada uno de los dominios evaluados en esta prueba. Se observa que Forma y Espacio, Números y Algebra presentan distribuciones relativamente simétricas; por su parte, el de Datos y Azar presenta una asimetría negativa, lo que revelaría un mayor dominio de la representación gráfica de los datos por parte de los sujetos.

FIGURA 1 - DISTRIBUCIÓN TOTAL DE RESPUESTAS SEGÚN DOMINIOS EVALUADOS PRUEBA DE HABILIDADES DE RAZONAMIENTO Y CONOCIMIENTO MATEMÁTICO

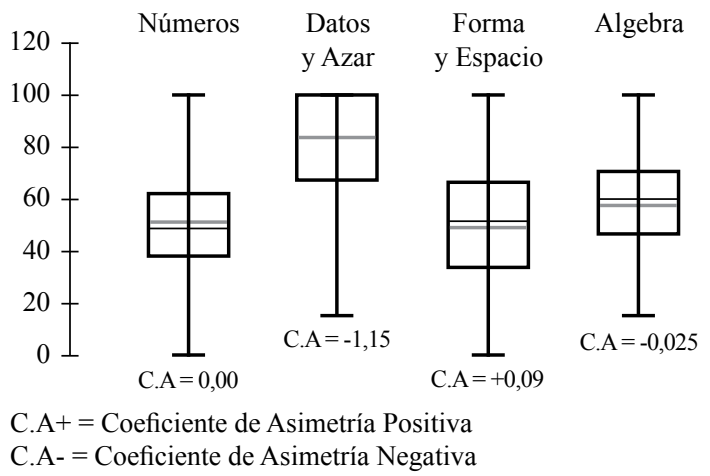

Por otra parte, es posible dar cuenta de diferencias a partir de la Institución de Educación Superior a la que pertenecen los estudiantes evaluados, pues al analizar la variabilidad de los resultados según universidad, se observa que ésta fluctúa entre $15,1 \%$ y $26,4 \%$ en la Universidad n. 2 ; y entre $19,6 \%$ y $26,2 \%$ en la 
Universidad n. 1. Dentro de ello resalta la Universidad n. 2, que presenta resultados más heterogéneos que la Universidad n. 1, en los dominios Datos y Azar y Algebra.

En este mismo sentido, al analizar la distribución de los puntajes de respuestas correctas por universidad (ver Figura 2), se aprecia que en todos los dominios evaluados los porcentajes de logro de la Universidad n. 2 presentan una distribución asimétrica negativa9; en cambio, en la Universidad n. 1 esto solo ocurre en Datos y Azar. Producto de lo anterior se puede observar que, en general, la Universidad n. 2 logra mayor porcentaje de respuestas correctas.

FIGURA 2 - DISTRIBUCIÓN TOTAL DE RESPUESTAS SEGÚN DOMINIOS EVALUADOS POR UNIVERSIDAD. PRUEBA DE HABILIDADES DE RAZONAMIENTO Y CONOCIMIENTO MATEMÁTICO

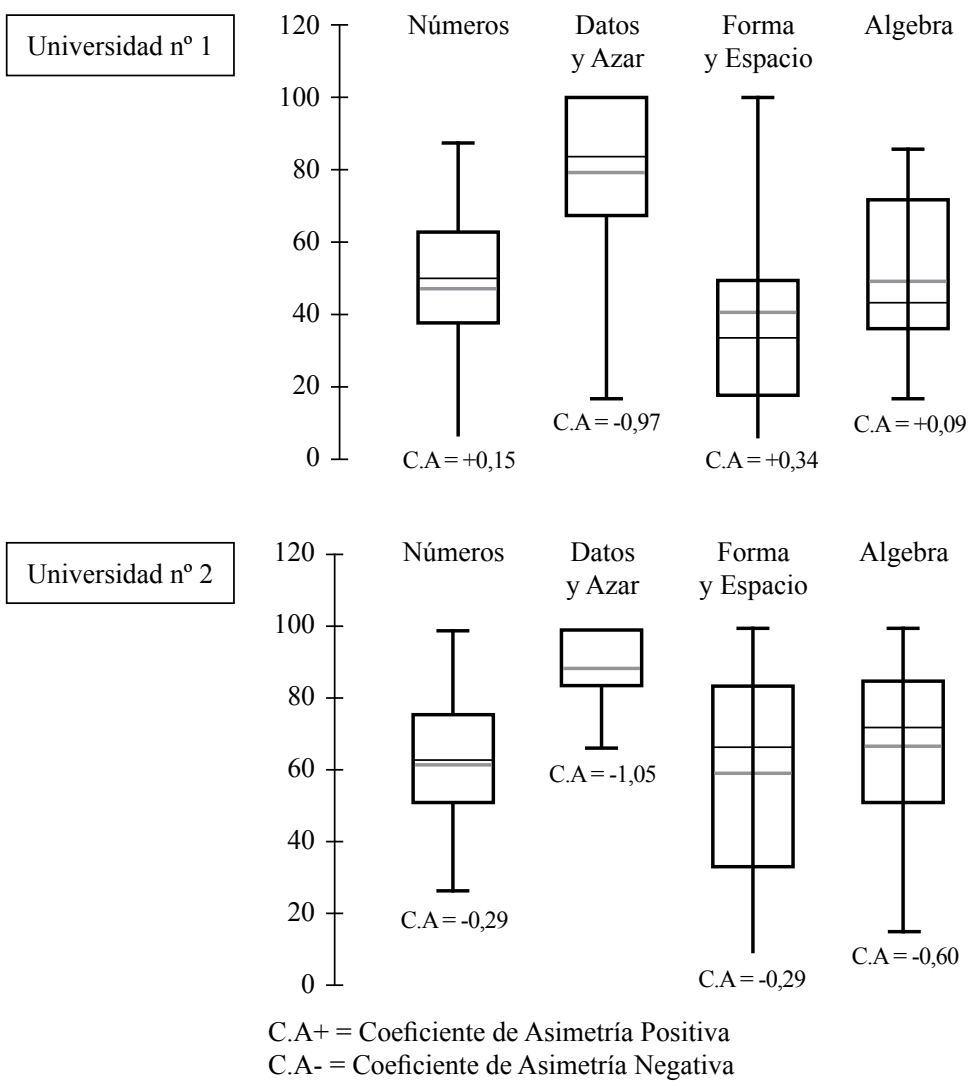

9 En todos los análisis de correlación se emplea la escala proporcionada por Saavedra (2005). 


\section{b) Prueba de Habilidades de Comprensión Lectora}

Los porcentajes de logro promedio en los cuatro dominios medidos en este instrumento se presentan en la Tabla 5. A partir de estos datos, es posible describir cuáles fueron aquellos de mayor y menor logro, así como comparar los resultados de los estudiantes evaluados según la Institución de Educación Superior a la que pertenecen.

TABLA 5 - PORCENTAJES DE LOGRO EN LOS DOMINIOS EVALUADOS SEGÚN INSTITUCIÓN. PRUEBA DE HABILIDADES DE COMPRENSIÓN LECTORA

\begin{tabular}{l|c|c|c|c}
\hline \multicolumn{1}{c|}{ DOMINIOS } & $\begin{array}{c}\mathbf{N}^{\mathbf{0}} \\
\text { Preguntas }\end{array}$ & $\begin{array}{c}\text { Universidad } \\
\mathbf{n}^{\mathbf{1}} \mathbf{1}(\mathbf{n = 3 0})\end{array}$ & $\begin{array}{c}\text { Universidad } \\
\mathbf{n}^{\mathbf{0}} \mathbf{2}(\mathbf{n = 5 0})\end{array}$ & $\begin{array}{c}\text { Total } \\
(\mathbf{n = 8 0})\end{array}$ \\
\hline Comprensión Literal & 9 & $78,9 \pm 20,1^{1}$ & $87,5 \pm 15,6$ & $84,3 \pm 17,8$ \\
\hline Comprensión Inferencial & 8 & $72,9 \pm 11,4$ & $83,5 \pm 12,7$ & $79,5 \pm 13,2$ \\
\hline Comprensión Critica & 9 & $72,2 \pm 17,9$ & $80,4 \pm 11,3$ & $77,4 \pm 14,6$ \\
\hline Reorganización de Información & 4 & $70,0 \pm 24,9$ & $84,5 \pm 22,5$ & $79,0 \pm 24,3$ \\
\hline
\end{tabular}

${ }^{1}$ : Promedio \pm Desviación Estándar (D.E).

En términos porcentuales generales, el nivel mejor logrado corresponde a Comprensión Literal con un 84,3\%; y el nivel más descendido es el de Comprensión Crítica, con un 77,4\%. A su vez, los mayores porcentajes de logro en todos los niveles medidos se observan en la Universidad n. 2, con puntajes promedios que fluctúan entre $80,4 \%$ y $87,5 \%$; en cambio en la Universidad n. 1 los puntajes promedios fluctúan entre $70,0 \%$ y $78,9 \%$.

En relación a la variabilidad de los resultados, el dominio que presenta respuestas más homogéneas es Comprensión Inferencial (D.E.=13,2), y el más heterogéneo corresponde a Reorganización (D.E.=24,3). Sin embargo, las desviaciones tienden a ser similares en ambas universidades en Comprensión Inferencial.

Por otra parte, se observa cómo es la distribución de las respuestas, tanto a nivel de grupo como por universidad. Al analizar la distribución general de los porcentajes de respuestas correctas, representadas en la Figura 3, por el total de la población en cada uno de los dominios evaluados, se observa que Comprensión Inferencial presenta una distribución relativamente simétrica; en cambio, los dominios de Comprensión Literal, Comprensión Crítica y Reorganización de Información presentan una distribución asimétrica negativa. En todos los dominios se presentan casos de respuestas atípicas. 
FIGURA 3 - DISTRIBUCIÓN TOTAL DE RESPUESTAS SEGÚN DOMINIOS EVALUADOS. PRUEBA DE HABILIDADES DE COMPRENSIÓN LECTORA

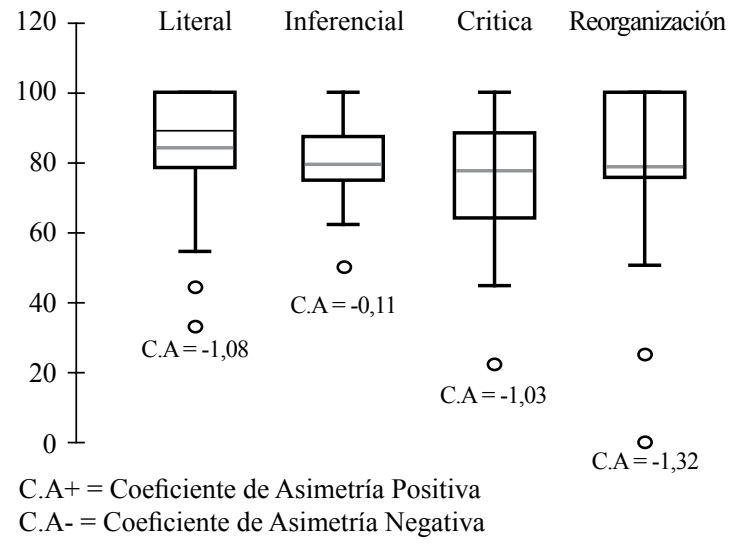

Al analizar los porcentajes de respuestas correctas por universidad, se observa que, en general, se presentan distribuciones similares, salvo en el dominio de Comprensión Inferencial; dado que es positiva en la Universidad n. 1 y negativa en la Universidad n. 2. Se observa, además, que en la Universidad n. 2 la distribución en los diferentes dominios de Comprensión Lectora es similar a la distribución general de la población evaluada (ver Figuras 3 y 4).

FIGURA 4 - DISTRIBUCIÓN TOTAL DE RESPUESTAS SEGÚN DOMINIOS EVALUADOS POR UNIVERSIDAD. PRUEBA DE HABILIDADES DE COMPRENSIÓN LECTORA

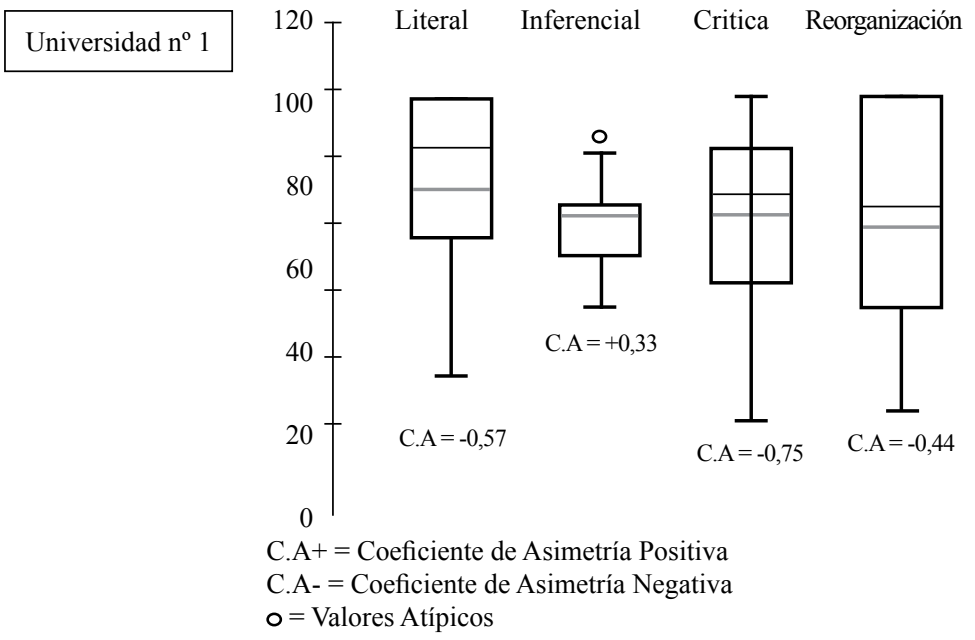


Universidad $\mathrm{n}^{\mathrm{o}} 2$

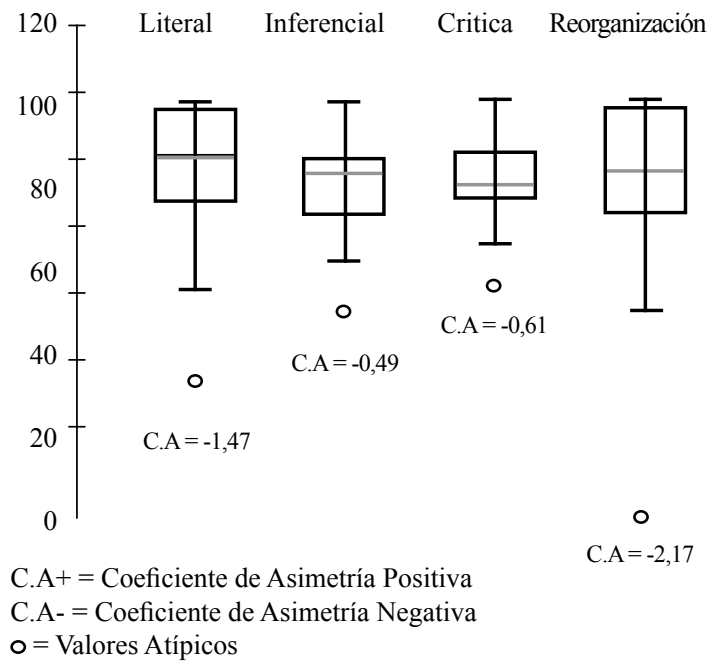

De las figuras anteriormente presentadas se desprende que los estudiantes logran mayores porcentajes de respuestas correctas en los dominios de Comprensión Literal, Comprensión Crítica y Reorganización de Información, independientemente de la universidad.

\section{Tipo de correlación entre dominios evaluados en cada instrumento y entre pruebas aplicadas}

a) Prueba de Habilidades de Razonamiento y Conocimiento Matemático

Al correlacionar el porcentaje de logro de las respuestas (Figura 5), se observa que existe una correlación positiva entre los cuatro dominios evaluados en este instrumento: Forma y Espacio, Datos y Azar, Algebra y Números. En particular, se observa una correlación positiva media entre Números y Algebra $(\mathrm{r}=0,60)$, Números y Forma y Espacio $(\mathrm{r}=0,54)$, Algebra y Datos y Azar $(\mathrm{r}=0,51)$ y Forma y Espacio y Algebra $(\mathrm{r}=0,51)$. Además, se desprende de la Figura 5 que la correlación entre el dominio Datos y Azar y los tres restantes es débil. 
FIGURA 5 - CÍRCULO DE CORRELACIONES DE LOS CUATRO DOMINIOS EVALUADOS. PRUEBA DE HABILIDADES DE RAZONAMIENTO Y CONOCIMIENTO MATEMÁTICO

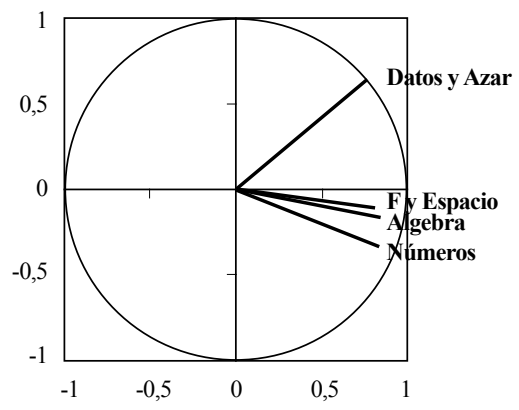

En la Figura 6 se muestra la correlación entre los porcentajes de logro según universidad, existiendo en ambos casos correlaciones positivas entre los dominios incluidos en esta evaluación. Específicamente, en la Universidad n. 1, Números y Forma y Espacio presentan una correlación positiva media $(\mathrm{r}=0,51)$, de manera similar Algebra y Datos y Azar $(\mathrm{r}=0,50)$, siendo en los demás dominios del tipo positivas débiles. En cambio, en la Universidad n. 2, Números y Algebra se correlacionan positivamente de manera media $(\mathrm{r}=0,63)$ y los dominios restantes presentan correlaciones débiles.

FIGURA 6 - CÍRCULO DE CORRELACIONES DE LOS CUATRO DOMINIOS EVALUADOS POR UNIVERSIDAD. PRUEBA DE HABILIDADES DE RAZONAMIENTO Y CONOCIMIENTO MATEMÁTICO

Universidad $\mathrm{n}^{\circ} 1$

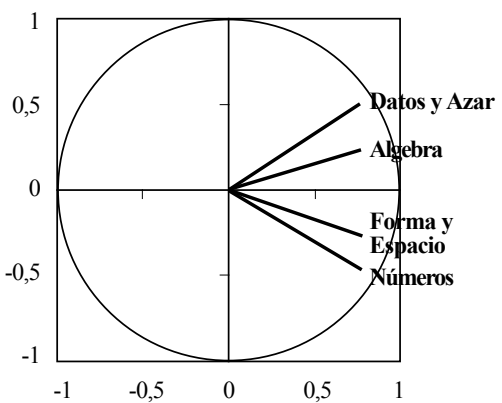

Universidad $\mathrm{n}^{\mathrm{O}} 2$

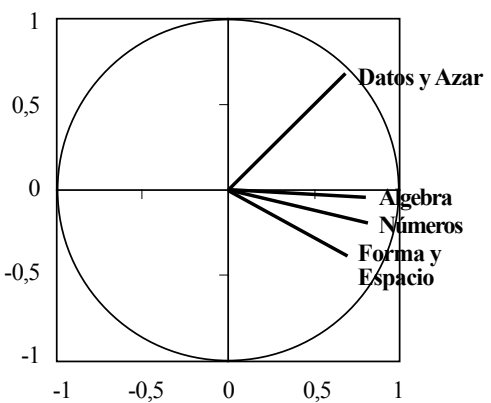




\section{b) Prueba de Habilidades de Comprensión Lectora}

Tal como se representa en la Figura 7, al correlacionar el porcentaje de logro general de la población entre los cuatro dominios evaluados en este instrumento, se observa una correlación positiva débil entre Comprensión Inferencial y Reorganización de Información $(\mathrm{r}=0,37)$ y entre Comprensión Literal y Reorganización de Información $(\mathrm{r}=0,35)$. También se desprende una baja correlación entre Comprensión Literal y Comprensión Crítica $(\mathrm{r}=0,10)$ y entre Comprensión Inferencial y Comprensión Crítica $(\mathrm{r}=0,15)$.

FIGURA 7 - CÍRCULO DE CORRELACIONES DE LOS CUATRO DOMINIOS EVALUADOS. PRUEBA DE HABILIDADES DE COMPRENSIÓN LECTORA

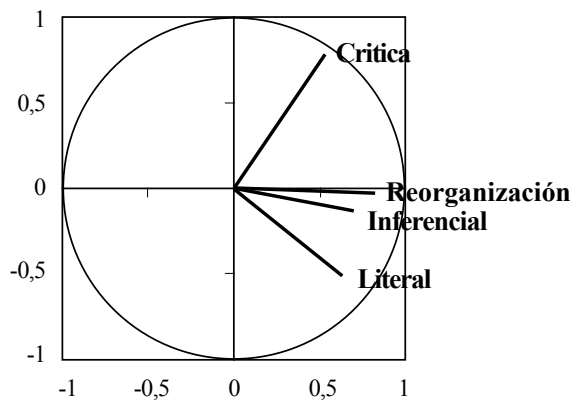

Por otro lado, tal como se representa en la Figura 8, al analizar las correlaciones por cada una de las universidades se observa que, en general, son débiles. Sin embargo, dentro de todos los dominios evaluados, se da una correlación similar entre la Comprensión Literal y Reorganización de Información en ambas universidades $(\mathrm{r}=0,30 \mathrm{y} \mathrm{r}=0,31)$, siendo a su vez las más altas de todas.

También se desprende que no hay coincidencia en cuanto a las correlaciones más débiles entre ambas universidades, ya que la Universidad n. 1 presenta una casi nula correlación inversa entre Comprensión Inferencial y Comprensión Literal $(\mathrm{r}=-0,05)$, en cambio, en la Universidad n. 2 la correlación entre Comprensión Literal y Comprensión Crítica es inversamente débil $(\mathrm{r}=-0,3)$. 
FIGURA 8 - CÍRCULO DE CORRELACIONES DE LOS CUATRO DOMINIOS EVALUADOS POR UNIVERSIDAD. PRUEBA DE HABILIDADES DE COMPRENSIÓN LECTORA

Universidad $n^{\circ} 1$

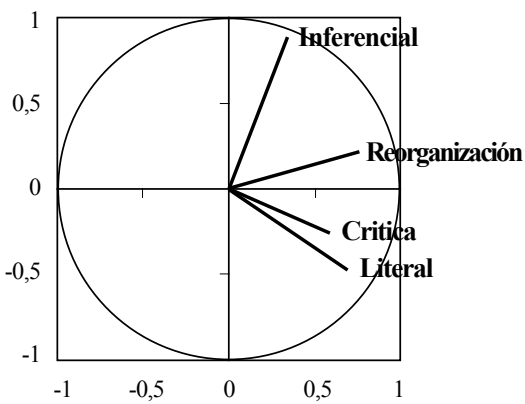

Universidad $\mathrm{n}^{\circ} 2$

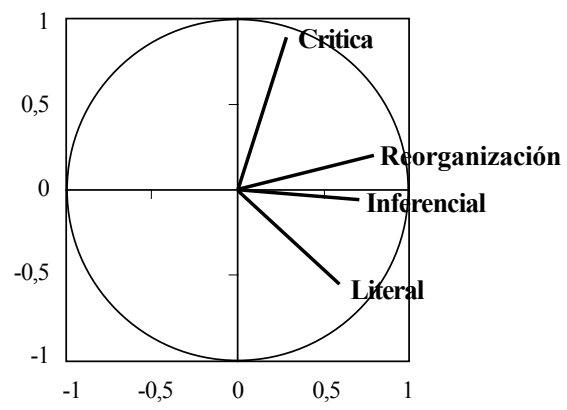

\section{Tipo de correlacion entre puntajes psu y los} dominios evaluados en los instrumentos

En general, se observan correlaciones positivas medias y débiles tanto entre los puntajes PSU Lenguaje y PSU Matemática, como entre los resultados obtenidos en las pruebas aplicadas en esta evaluación (ver Tabla 6). Sin embargo, dentro del conjunto de correlaciones, destaca la correlación de la PSU Lenguaje con el dominio de Comprensión Inferencial en la prueba de Habilidad de Comprensión Lectora $(\mathrm{r}=0,41)$. Al mismo tiempo, la correlación más débil con dicho instrumento se da con la Comprensión Crítica $(r=0,13)$. Por otro lado, en relación con los puntajes obtenidos en la PSU Matemática, destaca la correlación con el nivel de Reorganización de Información $(\mathrm{r}=0,41)$.

No obstante lo dicho anteriormente, ninguna de las pruebas PSU (Lenguaje y Matemática) podría explicar los resultados obtenidos en esta Prueba de Habilidad de Comprensión Lectora aplicada, dado que no se presentan correlaciones positivas fuertes, según la escala empleada (SAAVEDRA, 2005, p. 134). 
RUBILAR, P. R. S.; CARRILLO, M. F.; FUENTES, A. C. M.; ALVEAL, F. R. Evaluación de habilidades...

TABLA 6 - CORRELACIÓN PUNTAJES PSU Y LOS RESULTADOS OBTENIDOS EN LAS PRUEBAS APLICADAS POR CADA DOMINIO EVALUADO

\begin{tabular}{c|c|c|c|c}
\hline \multicolumn{4}{c}{ Prueba de Habilidades de Razonamiento y Conocimiento Matemático } \\
\hline $\begin{array}{c}\text { Dominios } \\
\text { Evaluados }\end{array}$ & Números & Datos y Azar & $\begin{array}{c}\text { Forma y } \\
\text { Espacio }\end{array}$ & Algebra \\
\hline PSU Matemática & 0,17 & 0,04 & 0,23 & 0,22 \\
\hline PSU Lenguaje & 0,42 & 0,27 & 0,46 & 0,53 \\
\hline
\end{tabular}

Prueba de Habilidades de Comprensión Lectora

\begin{tabular}{c|c|c|c|c}
\hline $\begin{array}{c}\text { Dominios } \\
\text { Evaluados }\end{array}$ & $\begin{array}{c}\text { Comprensión } \\
\text { Literal }\end{array}$ & $\begin{array}{c}\text { Comprensión } \\
\text { Inferencial }\end{array}$ & $\begin{array}{c}\text { Comprensión } \\
\text { Critica }\end{array}$ & $\begin{array}{c}\text { Reorganización } \\
\text { de Información }\end{array}$ \\
\hline PSU Matemática & 0,36 & 0,30 & 0,14 & 0,41 \\
\hline PSU Lenguaje & 0,31 & 0,41 & 0,13 & 0,28 \\
\hline
\end{tabular}

Por otro lado, en la tabla anterior, se observa una correlación positiva media entre los puntajes PSU Lenguaje y los resultados obtenidos en el dominio Álgebra $(\mathrm{r}=0,53)$, siguiéndolo muy de cerca Forma y Espacio $(\mathrm{r}=0,46)$ y Números $(\mathrm{r}=0,42)$.

Se destaca la escasa correlación lineal entre los puntajes obtenidos en la PSU Matemática y el dominio Datos y Azar $(\mathrm{r}=0,04)$, puesto que, además, en relación con la PSU Lenguaje se presenta con un valor de correlación más bajo, comparativamente a los otros dominios $(\mathrm{r}=0,27)$.

Dentro de la evaluación realizada, al comparar los puntajes obtenidos en la PSU (Lenguaje y Matemática) con los resultados de la Prueba de Habilidades de Razonamiento y Conocimiento Matemático aplicada, se desprende que los valores de las correlaciones son, en general, más débiles en la PSU Matemática que en la PSU Lenguaje.

Finalmente, y en coherencia con lo expresado en el análisis anterior, si bien no se puede afirmar que los puntajes PSU de los estudiantes evaluados, en general, pueden explicar los resultados obtenidos en las pruebas aplicadas (especialmente la PSU Matemática), se puede afirmar que existiría una tendencia a explicar el logro obtenido en el dominio Álgebra, por los resultados de la PSU Lenguaje, con una correlación media $(\mathrm{r}=053)$. 


\section{Discusión y conclusiones de la evaluación}

Si bien la muestra corresponde a dos instituciones de Educación Superior del Consejo de Rectores, esta presenta algunas similitudes con las características de la población de estudiantes de Pedagogía. Así pues, a nivel nacional, el $70 \%$ de los estudiantes son de género femenino (la muestra corresponde a $79 \%$ ), además, según el informe de la prueba diagnóstica de los estudiantes de pedagogía que egresan de la universidad, el $42 \%$ de ellos proviene de establecimientos Municipales y 47 a Particulares Subvencionado, siendo la muestra de estudio de 44 y 56 respectivamente (MINEDUC, 2010, p. 28; MIZALA et. al., 2000).

Por lo anterior, si bien la muestra del estudio no es representativa a nivel nacional, las similitudes de ella con la población nacional, permite que las presentes conclusiones puedan ser transferidas al resto de los estudiantes en Formación Inicial Docente, al menos a modo de hipótesis plausibles; especialmente, en aquellas Instituciones Formadoras de Regiones distintas a la Metropolitana ${ }^{10}$ que también pertenecen al Consejo de Rectores de Chile.

Ahora bien, las presentes conclusiones darán cuenta, por un lado, de las principales debilidades detectadas, las que deben ser subsanadas en la Formación Inicial Docente por parte de las instituciones formadoras, sino que además se propondrán sugerencias a partir de algunos hallazgos relevantes:

a) Principales debilidades develadas por la evaluación en Habilidades de Razonamiento y Conocimiento Matemático y Comprensión Lectora:

- Sobre las Habilidades de Razonamiento y Conocimiento Matemático:

Del análisis de los resultados en la prueba es posible señalar que el dominio más descendido es Forma y Espacio. Lo anterior se podría deber al hecho de que Geometría no ocupa un lugar preponderante en los contenidos enseñados por parte de los profesores, a pesar de estar en el currículo en Educación Básica y Media, así al menos lo señala el estudio de Espinoza et. al. (2007, p. 2):

La geometría es en Chile, y muy probablemente en varios países del mundo, una de las áreas de la matemática que presenta mayores dificultades para ser enseñada y aprendida [...] Nuestra investigación, y otros estudios realizados en este ámbito (Espinoza, Barbé,

10 Chile es un Estado Unitario, que se divide políticamente en Regiones. Siendo la Región Metropolitana la que concentra el poder político y económico, ubicándose, por lo mismo, la capital de Chile Santiago. 
2004; OCDE, 2004), muestran que los profesores de estos niveles educativos, debido a su escasa formación matemática y didáctica, tienen serias dificultades para ir más allá de una enseñanza de la geometría centrada en la clasificación rígida y formal de figuras y cuerpos, en la memorización de propiedades y en el cálculo de áreas y perímetros. Por ello, tienden frecuentemente a postergar la enseñanza de la geometría para finales del año escolar, terminando por enseñarla superficialmente por cuestiones de tiempo y disponibilidad en las escuelas.

Los estudios del MINEDUC plantean algo similar, al constatar que si bien en el currículo formalmente se explicita la enseñanza de los cuatro ejes señalados anteriormente, los tiempos dedicados por parte de los profesores a la enseñanza en el eje de Geometría es notoriamente menor:

[...] las Operaciones Aritméticas y los Números Naturales son los contenidos predominantes. Los restantes contenidos (Geometría, Orientación en el espacio, Fracciones y Resolución de Problemas) reciben una dedicación de tiempo notoriamente menor (MINEDUC, 2004, p. 11).

En todo caso, los resultado de la presente evaluación son coherentes con el informe derivado de la medición TIMSS (2003), en el cual Geometría es uno de los dominios con niveles de logro más descendidos. Considerando que las conclusiones del TIMSS corresponden a una población distinta, esto adquiere relevancia, puesto que los estudiantes que ingresan a estudiar Pedagogía en Educación General Básica mantendrían dicha debilidad, constatada en los resultados de esta evaluación.

Por otro lado, los dominios con mayores porcentajes de respuestas correctas son Números y Algebra; probablemente debido a que, en general, los profesores, al plantear problemas de tipo numérico y en Álgebra, tienden a privilegiar el uso de fórmulas más que las deducciones de las mismas. Además, Datos y Azar presenta un dominio similar al de Álgebra, asunto que podría explicarse en el hecho de que uno de los contenidos y habilidades más frecuentemente desarrolladas en Estadística es la representación de datos a través de gráficos de barras y pictogramas, que son coherentes con los tipos de ítems evaluados en este instrumento. 
Por otra parte, al analizar la correlación entre los porcentajes de logro de las respuestas a nivel general se observa una correlación positiva media entre algunos dominios. Estos son Números y Algebra $(\mathrm{r}=0,60)$, Número y Forma y Espacio $(\mathrm{r}=0,54)$. Ello puede deberse a que en el Sistema Escolar (tanto en el ciclo de Educación Básica como en el de Educación Media), en el sector de Educación Matemática, los alumnos trabajan los contenidos de Algebra, Forma y Espacio como una cuestión eminentemente numérica. Por ejemplo, en Algebra se privilegia la aplicación de fórmulas o despejar ecuaciones de primer grado; y en Forma y Espacio se insta a resolver problemas como operatoria numérica y/o aplicación de formulas. En otras palabras, ambos dominios (Algebra, Forma y Espacio) tienden a ser asimilados como mera cuestión de cálculo y de procedimiento numérico; por ello se puede comprender su alta correlación con el eje Número.

\section{- Sobre la Habilidad de Comprensión Lectora:}

Como se mencionó anteriormente, el nivel mejor logrado es Comprensión Literal en ambas universidades. Este resultado se puede atribuir a dos factores. En primer lugar, al hecho de que dicho nivel dominio es el que representa menor grado de dificultad en las evaluaciones, dado que las respuestas se encuentran explícitamente en el texto, y en segundo lugar, a que dicha habilidad es la más desarrollada en el Sistema Escolar, especialmente en el ciclo de Educación Básica.

Por su parte, el nivel más descendido, en general, corresponde al de Comprensión Crítica. Esto podría explicarse por ser la menos desarrollada en el Sistema Escolar y, a su vez, por el hecho de implicar una interpretación personal a partir del texto, lo que supone un mayor grado de complejidad (CATALÁ et. al., 2005).

Ello es coherente, en sentido general, con el hallazgo del informe derivado de la medición PISA (2000), que da cuenta que los alumnos del Sistema Escolar evaluados presentan una aceptable capacidad en tareas básicas de lectura, lo que implicaría que tienen bajos niveles de logro en tareas de lectura más complejas, tales como las involucradas para alcanzar el nivel de Comprensión Crítica. Considerando que la prueba PISA corresponde a una población de estudio distinta, esto adquiere relevancia, puesto que los estudiantes que ingresan a estudiar Pedagogía en Educación General Básica mantienen mejores niveles de logro en Comprensión Literal, no así en los niveles que suponen mayor grado de complejidad, tal como se constata en los resultados de esta evaluación.

Al realizar un análisis de correlación entre los diferentes niveles evaluados, los mayores valores corresponden al de Reorganización de Información con Comprensión Inferencial $(r=0,37)$ y Comprensión Literal $(r=0,35)$. Ello puede deberse a que en el Sistema Escolar, especialmente en el sector de Lenguaje 
y Comunicación, los alumnos trabajan permanentemente la lectura y habilidades de completar y reogarnizar la información del texto desde el ciclo de enseñanza básica.

Además, se observa que la correlación más baja se produce entre el nivel de Comprensión Literal y Comprensión Crítica $(\mathrm{r}=0,10)$. Una explicación plausible podría estar en que ambos son aprendizajes de distinta naturaleza, puesto que el primero supone identificar la información explícita en el texto, en cambio, el segundo, implica analizar la información entregada por el texto con información obtenida por el estudiante en otras fuentes o saberes de su capital cultural, en virtud de la interpretación y la formulación de juicios sobre el texto.

Finalmente, en conclusión, se espera que estas debilidades (tanto las de Razonamiento y Conocimiento Matemático como las de Comprensión Lectora) sean subsanadas en la formación de los futuros docentes.

b) Desafíos para la Formación Inicial Docente por parte de las Instituciones Formadoras:

- Incorporación de actividades curriculares formales que favorezcan el desarrollo de ambas habilidades evaluadas:

$\mathrm{El}$ análisis de los datos evidencia un bajo nivel de logro en ambas pruebas considerando que evalúan, en general, habilidades que deberían desarrollarse en los ciclos de formación de la Educación Básica y Media de nuestro Sistema Escolar; además, comparativamente, las Habilidades de Conocimiento y Razonamiento Matemático son más descendidas que la Habilidad de Comprensión Lectora.

De lo anterior, se puede inferir que los estudiantes pertenecientes a las universidades en este caso ingresan con las Habilidades de Razonamiento y Conocimiento Matemático y Comprensión Lectora por debajo de lo esperado. Esta situación implica un desafío para las Instituciones Formadoras, que debieran potenciar el desarrollo de estas habilidades y asegurar su logro, en niveles superiores de desempeño, al finalizar el proceso de Educación Terciaria en el nivel de pregrado.

Concretamente, se hace necesario que las Instituciones Formadoras incorporen, en sus planes y programas de estudios, aprendizajes y contenidos asociados al desarrollo de las habilidades aquí evaluadas, especialmente en las actividades curriculares referidas a la formación en los sectores de Educación Matemática y Lenguaje y Comunicación, puesto que se espera que estas habilidades sean aprendidas por todos los estudiantes de las carreras de Educación General Básica para ser enseñadas posteriormente en el Sistema Escolar, tal como lo expresa el Marco Curricular vigente y la propuesta de Ajustes Curriculares en proceso de implementación a partir del año 2010. 
- Incorpora'ción en el Plan de Estudio de actividades curriculares formales, que favorezcan específicamente el desarrollo de la Habilidad de Comprensión Lectora de los futuros docentes:

$\mathrm{Al}$ analizar las correlaciones entre la PSU Matemática y PSU Lenguaje con los resultados de la evaluación en ambas pruebas son en general débiles, se destaca el valor entre la PSU Lenguaje y el eje Algebra $(r=0,53)$, así como entre ésta y el eje Forma y Espacio $(\mathrm{r}=0,46)$ con una correlación directa. De esto, se infiere que los resultados de la PSU Lenguaje tendrían una mayor incidencia en los logros obtenidos en la evaluación de Habilidades de Razonamiento y Conocimiento Matemático, a través del instrumento aplicado, tanto a nivel general como en los dominios antes mencionados, de forma particular.

Lo anterior, se puede relacionar con el hecho de que la formulación de los reactivos para evaluar los distintos dominios (Número, Datos y Azar, Álgebra, Forma y Espacio) se caracteriza por incluir en su presentación enunciados de tipo lingüístico. Así, es necesario leer, organizar e identificar los elementos claves de los problemas, para su resolución desde una perspectiva matemática. En otras palabras, supone organizar (o reorganizar) la información e inferir del texto el problema matemático para dar una respuesta. En este sentido, ello también explicaría que la correlación más alta entre la PSU Matemática se dé con el nivel de Reorganización de la Información ( $\mathrm{r}=0,41)$.

Cabe señalar que estas relaciones entre la Comprensión Lectora y el Razonamiento y Conocimiento Matemático se encuentran esbozadas en los informes de las pruebas TIMSS (2003), en los cuales se afirmaba que los estudiantes ahí evaluados sólo podían reconocer problemas cuando la formulación matemática estaba explícita en el texto (lo que en esta evaluación se operacionaliza como Comprensión Literal). En dicho informe, además, se afirma que nuestros alumnos no podían identificar ni resolver problemas que requieren interpretar y formular un problema en términos matemáticos (que para este caso se asocia a los dominios de Reorganización de la Información y Comprensión Inferencial).

De esta forma, asumiendo la existencia de una relación entre Comprensión Lectora y las habilidades de Razonamiento y Conocimiento Matemático, se puede sostener como hipótesis que a mayor resultado de la PSU Lenguaje mejores resultados en la resolución de problemas en los distintos dominios matemáticos evaluados en esta ocasión. Ahora bien, si la PSU Lenguaje se construye bajo el supuesto de medir Comprensión Lectora, se puede inferir que mejorando los niveles de dominio de la misma, se mejorará el desarrollo de las Habilidades de Razonamiento y Conocimiento Matemático.

De todo el análisis anterior, se puede inferir que mejorando la Habilidad de Comprensión Lectora de los estudiantes de Pedagogía Básica, es posible mejorar ciertos dominios en el razonamiento matemático y, considerando que 
dichos profesores, cuando ejerzan tendrán en ambos sectores que dedicar más del la mitad de su tiempo de enseñanza, se hace razonable que las instituciones formadoras tengan actividades curriculares formales para mejorar la comprensión lectora y con ello, además, mejorar potencialmente el razonamiento matemático, especialmente lo referido a resolución de problemas (que en el actual marco curricular aparece como aprendizaje clave o central en matemática).

Finalmente, en coherencia con el postulado anterior, se esperaría que en la Formación Inicial Docente de los profesores de Educación General Básica, con o sin mención, fortalezcan los niveles de Comprensión Lectora no sólo para asegurar un buen desempeño futuro en el sector de Lenguaje y Comunicación, sino que, por sobretodo, para asegurar la adquisición de las habilidades fundamentales para la enseñanza de la Matemática como lo son el Razonamiento y Conocimiento Matemático.

c) Desafíos para las Políticas Públicas en Educación:

Considerando que uno de los principales problemas a resolver en esta evaluación se refiere a que no existen estándares nacionales normalizados referidos a las habilidades de Razonamiento y Conocimiento Matemático y Comprensión Lectora, tanto para el ingreso como para el egreso de la Carrera de Pedagogía en Educación General Básica en Chile, tal cual se señaló en el punto "Tipo de Evaluación y Análisis de Datos"; es recomendable que las autoridades encargadas de diseñar las Política Publicas en Educación, especialmente las referidas a la Formación Inicial Docente, puedan definir estándares mínimos normalizados, ya sea para el ingreso a la formación profesional o su habilitación para el ejercicio profesional, que asegure que las habilidades a enseñar sean dominadas por los profesores, dado que no se puede enseñar lo que no se conoce o posee.

\section{REFERENCIAS}

CATALÁ, G. et. al. Evaluación de la comprensión lectora. Pruebas ACL (1. ${ }^{\circ}$ 6. ${ }^{\circ}$ de primaria). España: Ed. Graó, 2005.

COLLIN, A. Introduction to Multivariate Analysis. Chapman and Hall. Cambridge. Great Britain, 1992.

COOK, T.; REICHARDT, Ch. Métodos cualitativos y cuantitativos en investigación evaluativa. España: Ed. Morata S.L., 1986. 
CRIVISQUI, E. Análisis factorial de correspondencias. Paraguay: Editorial de la Universidad Católica de Asunción, 1993.

EDUCARCHILE. Sobre la beca de inscripción y los quintiles. 2010. Disponible en: $<$ http://www.educarchile.cl/Portal.Base/Web/VerContenido.aspx?GUID=1 23.456.789.000\&ID=106191>. Consultado en: 25/5/2010.

ESPINOZA et. al. El problema de la enseñanza de la geometría en la Educación General Básica chilena y una propuesta para su enseñanza en aula. 2007. Disponible en: <http://www4.ujaen.es/ aestepa/TAD_II/Comunicaciones_ TAD_II/34\%20-\%20Espinoza_Barbe_congres_TAD_2.pdf $>$.

EYZAGUIRRE, B.; FONTAINE, L. ¿Qué mide realmente el SIMCE? 1999. Disponible en: $<$ http://www.simce.cl/fileadmin/publicaciones-BD-simce/rev75_ eyzaguirre.pdf $>$. Consultado en: 23/5/2010.

. Las escuelas que tenemos. Santiago de Chile: Centro de Estudios Públicos (CEP), 2008.

GARCÍA-HUIDOBRO, J.; CÓX, C. La reforma educacional chilena 19901998, visión de conjunto. En: GARCÍA-HUIDOBRO, J. (Ed.). La reforma educacional chilena. España: Ed. Popular, 1999.

JONSON, R.; WICHERN, D. Applied multivariate statitical analysis. New Jersey, U.S.A.: Prentice Hall Englewood Cliffs, 1988.

LATORRE, A. et al. Bases metodológicas de la investigación educativa. España: Ed. Hurtado, 1997.

LLECE. Primer Estudio Internacional Comparativo sobre Lenguaje, Matemática y Factores Asociados en Tercero y Cuarto Grado. Laboratorio Latinoamericano de Evaluación de Calidad de la Educación. Santiago, Chile: UNESCO, 1998. Disponible en: $<$ http://www.oei.es/quipu/primer_informeLLECE.pdf $>$. Consultado en: $14 / 4 / 2009$.

. Primer Estudio Internacional Comparativo sobre Lenguaje, Matemática y Factores Asociados para alumnos del tercer y cuarto grado de la Educación Básica. Laboratorio Latinoamericano de Evaluación de Calidad de la Educación. Santiago, Chile: UNESCO, 2000. Disponible en: <http://www.oei.es/quipu/ segundo_informeLLECE.pdf $>$. Consultado en: 5/5/2009.

LLINARES, S. Intentando comprender la práctica del profesor de matemáticas. En: DA PONTE, J. P.; SERRAZINA, 1 (Coord.). Educação Matemática em 
Portugal, Espanha e Italia. Lisboa, Portugal: Secção de Educação Matemática da Sociedade Portuguesa de Ciêncas de Educação, 2000.

MINEDUC. Objetivos Fundamentales y Contenidos Mínimos Obligatorios para la Educación General Básica y normas generales para su aplicación. Decreto Ley n. 40, del 24-01-96, Santiago de Chile. 1996.

. Objetivos Fundamentales y Contenidos Mínimos Obligatorios para la Educación Media. Decreto Ley n. 220, Santiago de Chile. 1998.

. Bases curriculares de la Educación Parvularia. Ministerio de Educación de Chile. 2001. Disponible en: <http://www.oei.es/inicial/curriculum/bases_ curriculares_chile.pdf $>$. Consultado en: 20/5/2009.

. Implementación Curricular en el Aula: Matemáticas Primer Ciclo Básico. Seguimiento a la Implementación Curricular; Unidad de Currículum y Evaluación. Ministerio de Educación de Chile. 2004. Disponible en: $<$ http://www. curriculum-mineduc.cl/docs/estudio/cobertura_curricular_en_matematicas_1_ ciclo.pdf $>$.

. Mapas del Progreso del Aprendizaje. Sector Matemática Mapa de Progreso de Números y Operaciones. Ministerio de Educación de Chile. 2007. Disponible en: $<$ http://www.curriculum-mineduc.cl/docs/mapas/mapa-matematica. pdf $>$. Consultado en: 25/6/2009.

.Propuesta ajustes curriculares: Objetivos Fundamentales y Contenidos Mínimos Obligatorios Matemática. Ministerio de Educación de Chile. 2009a. Disponible en: $<$ http://www.curriculum-mineduc.cl/ayuda/docs/ajuste-curricular-2/ Ajuste_Matematica_300309.PDF>.Consultado en: 25/6/2009.

. Fundamentos del Ajuste Curricular en el sector de Lenguaje y Comunicación. Ministerio de Educación de Chile. 2009b. Disponible en: $<$ http://www.curriculum-mineduc.cl/ayuda/docs/ajuste-curricular-2/Articulo_ Fundamentos_Ajuste_Lenguaje_300309.pdf $>$. Consultado en: 25/6/2009.

. Programa Inicia. 2010. Disponible en:: <http://www.programainicia.

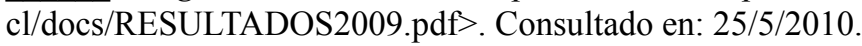

. Programa Inicia. Ministerio de Educación de Chile. Santiago, Chile. Disponible en: <http://www.programainicia.cl/>. Consultado en: 25/6/2009.

MIZALA, A. et. al. Los Maestros en Chile: Carreras e Incentivos. 2000. En: Inter-American Development Bank, Banco Interamericano de Desarrollo, 
Latin American Research Network, Red de Centros de Investigación. Research Network Working paper \#R-403. Disponible en: $<$ http://idbdocs.iadb.org/wsdocs/ getdocument.aspx?docnum=788031>. Consultado en: 25/5/2010.

PISA. Competencias para la vida. Resultados de los estudiantes chilenos en el estudio PISA 2000. Program for International Student Assesment y Ministerio de Educación de Chile. 2000. Disponible en: <http://www.simce.cl/fileadmin/ Documentos_y_archivos_SIMCE/evaluaciones_inter/pisa_2000/informe_ CHILE_PISA_2000.pdf $>$. Consultado en: 20/6/2009.

QUIROZ, R. Obstáculos para la apropiación del contenido académico en la escuela secundaria. Infancia y Aprendizaje, España, n. 55, p. 45-58, 1991.

SAAVEDRA, E. Contenidos básicos de Estadística y Probabilidad. Chile: Ed. Universidad de Santiago, 2005.

SANDOVAL, P. Políticas de descentralización curricular y autonomía profesional: contradicciones y posibilidades en el caso chileno. Reflexão e Ação, Brasil, v. 7, p. 93-106, 1999.

SIMCE. Informe de resultados nacionales 2008. Sistema de Medición de la Calidad de la Educación, Ministerio de Educación de Chile. 2008. Disponible en: <http://simce.cl/fileadmin/Documentos_y_archivos_SIMCE/Informes Resultados_2008/Informe_Nacional_2008.pdf $>$. Consultado en: 25/3/2009.

TIMSS. Chile y el aprendizaje de Matemáticas y ciencias según TIMSS. Resultados de los estudiantes de $8 .^{\circ}$ básico en el estudio Internacional de Tendencias en Matemáticas y Ciencias 2003. International Association for the Evaluation of Educational Achievement (IEA) y Ministerio de Educación de Chile. 2003. Disponible en: <http://www.simce.cl/index.php?id=103\&no_ cache $=1>$. Consultado en: 25/6/2009.

Texto recebido em 13 de abril de 2010 .

Texto aprovado em 28 de maio de 2010. 